\title{
THEOREM ON MAGNET FRINGE FIELD
}

\author{
JIE WEI $^{1}$ and RICHARD TALMAN ${ }^{2}$ \\ ${ }^{1}$ Relativistic Heavy Ion Collider, Brookhaven National Laboratory, \\ Upton, New York 11973, USA \\ ${ }^{2}$ Laboratory of Nuclear Studies, Cornell University, Ithaca, New York 14853, USA
}

(Received 2 January 1996; in final form 2 January 1996)

\begin{abstract}
The standard transverse magnetic field multipole expansion strictly describes field integrals with integration range starting well before and ending well after the magnet. This paper shows that in all practical cases, for non-solenoidal magnets, a similar expansion (with no longitudinal field components) gives a highly accurate description of the deflection suffered at a single end, that is, as a particle travels from well inside to well outside the magnet.
\end{abstract}

Keywords: Fringe field.

Transverse particle motion in particle accelerators is governed almost totally by non-solenoidal magnets for which the body magnetic field can be expressed as a series expansion of the normal $\left(b_{n}\right)$ and skew $\left(a_{n}\right)$ multipoles,

$$
B_{y}+i B_{x}=\sum_{0}^{\infty}\left(b_{n}+i a_{n}\right)(x+i y)^{n},
$$

where $x, y$, and $z$ denote horizontal, vertical, and longitudinal (along the magnet) coordinates. Since the magnet length $L$ is necessarily finite, deflections are actually proportional to "field integrals" such as $\bar{B} L \equiv$ $\int B(x, y ; z) d z$ where the integration range starts well before the magnet and ends well after it. For $\bar{a}_{n}, \bar{b}_{n}, \bar{B}_{x}$, and $\bar{B}_{y}$ defined this way, the same expansion Equation 1 is valid and the "standard" approximation is to neglect any deflections not described by this expansion, in spite of the fact that Maxwell's equations demand the presence of longitudinal field components at the magnet ends. The purpose of this note is to provide a semi-quantitative estimate of the importance of $\left|\Delta p_{\|}\right|$, the transverse deflection produced by 

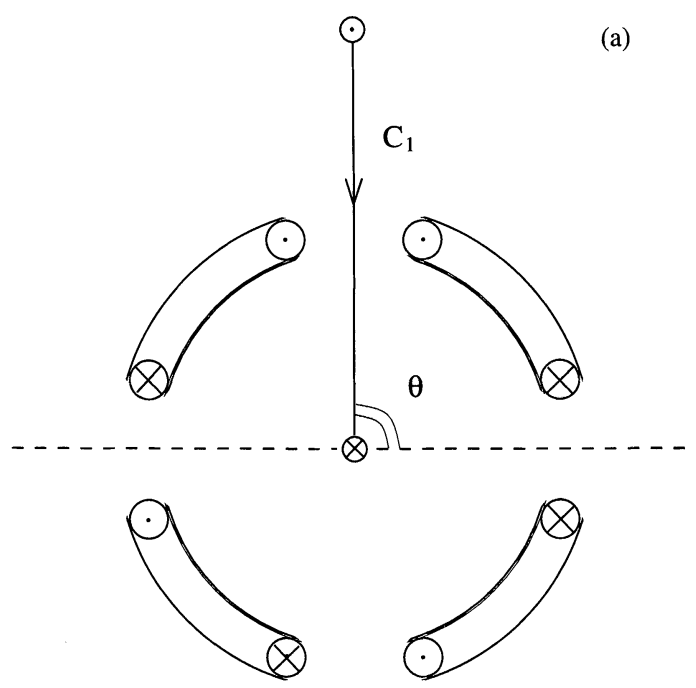

(b)

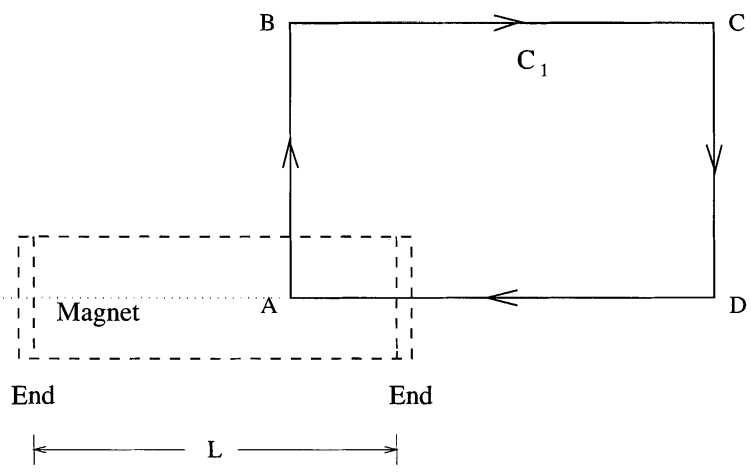

FIGURE 1 (a) Front and (b) side view of the closed curve $C_{1}$ of integration extending from inside the body of the magnet to infinity without enclosing any electric current.

the longitudinal component of the fringe field at one magnet end relative to $\left|\Delta p_{0}\right|$, the total deflection produced by passage through the whole magnet. To emphasize the generality and simplicity of the result it is given in the form of a theorem. The essence of the proof is an evaluation of the contribution 
of the longitudinal field $B_{z}$ from the vicinity of one magnet end since, along a path parallel to the magnet axis such as path BC in Figure 2, there is no contribution to the longitudinal field integral either from well inside or well outside the magnet.

THEOREM For any non-solenoidal magnet,

$$
\left\langle\frac{\left|\Delta p_{\|}\right|}{\left|\Delta p_{0}\right|}\right\rangle \approx \frac{\epsilon_{\perp}}{L} \sqrt{\frac{1+0.75 \beta^{\prime 2}}{8}} \approx \frac{\epsilon_{\perp}}{L},
$$

where $\epsilon_{\perp}$ is the rms beam transverse emittance, $\beta^{\prime}=d \beta / d s,\langle\rangle$ denotes the average over betatron phase, and the final approximation must be withdrawn if $\beta^{\prime}$ is anomalously large.

Proof According to the principle of linear superposition, the fringe field at the end of the magnet can, in accordance with Equation 1, be written as

$$
\mathbf{B}_{F}=\sum_{0}^{\infty}\left(\mathbf{B}_{F n}+\mathbf{A}_{F n}\right)
$$

where $B_{F n}$ (or $A_{F n}$ ) is the fringe field that corresponds to $b_{n}$ (or $a_{n}$ ).

As shown in Figures 1a and $\mathrm{b}$, for any multipole $b_{n}$ or $a_{n}$, there exists a closed curve $C_{1}$ extending from inside the body of the magnet to infinity without enclosing any electric current. The cross angle $\theta$ between the plane containing curve $C_{1}$ and the horizontal plane is chosen to be

$$
\theta= \begin{cases}\frac{2 k+1}{2(n+1)} \pi, & \text { for } a_{n} \\ \frac{k+1}{n+1} \pi, & \text { for } b_{n},\end{cases}
$$

where $0 \leq k<n$ is an integer, so that

$$
\int_{A}^{B} \mathbf{B} \cdot d \mathbf{l}=0
$$




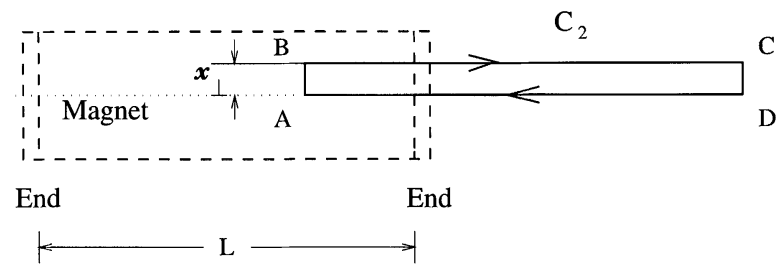

FIGURE 2 Side view of the closed curve $C_{2}$ for integration extending from inside the body of the magnet to infinity without enclosing any electric current.

Because the magnet field at infinity is equal to zero, one obtains from Ampere's law,

$$
\int_{D}^{A} B_{F n, z}(x=y=0 ; z) d z=\int_{D}^{A} A_{F n, z}(x=y=0 ; z) d z=\int_{D}^{A} \mathbf{B} \cdot d \mathbf{l}=0 .
$$

Hence,

$$
\int_{D}^{A} B_{z}(x=y=0 ; z) d z=0 .
$$

Suppose that the particle moves across the end of the magnet at a transverse displacement $x_{\perp}$, as shown by the trajectory BC in Figure 2. Using Equation 7 and the closed curve of integration $C_{2}$, we obtain

$$
\int_{B}^{C} B_{z}\left(x_{\perp}\right) d z=-\int_{A}^{B} \mathbf{B}_{\perp}\left(\mathbf{x}_{\perp}^{\prime}\right) \cdot d \mathbf{x}_{\perp}^{\prime}
$$

Hence, the transverse deflection $\Delta p_{\|}$produced by the longitudinal component of the fringe field is 


$$
\left|\Delta p_{\|}\right|=e\left|\int_{B}^{C} \mathbf{v}_{\perp} \times \mathbf{B}_{z}\left(x_{\perp}\right) d z\right|=e\left|v_{\perp} \int_{A}^{B} \mathbf{B}_{\perp}\left(\mathbf{x}_{\perp}^{\prime}\right) \cdot d \mathbf{x}_{\perp}^{\prime}\right| \approx e\left|v_{\perp} x_{\perp} B_{\perp}\left(\mathbf{x}_{\perp}\right)\right| .
$$

The total deflection $\Delta p_{0}$ produced by the main magnet body is

$$
\left|\Delta p_{0}\right|=e\left|\int_{0}^{L} \mathbf{v}_{z} \times \mathbf{B}_{\perp} d z\right| .
$$

The factor $v_{\perp} x_{\perp}$ can be averaged as follows. By the standard "pseudoharmonic" description of betatron motion, letting $S=\sin \psi, C=\cos \psi$,

$$
x=\sqrt{\epsilon_{\perp} \beta} C, \quad x^{\prime}=\sqrt{\frac{\epsilon_{\perp}}{\beta}}\left(S-C \frac{\beta^{\prime}}{2}\right) .
$$

Using the results $\left\langle C^{2} S^{2}\right\rangle=1 / 8,\left\langle C^{4}\right\rangle=3 / 8$, and $\left\langle C^{3} S\right\rangle=0$, one obtains

$$
\sqrt{\left\langle x^{2} x^{\prime 2}\right\rangle}=\epsilon_{\perp} \sqrt{\frac{1+0.75 \beta^{\prime 2}}{8}},
$$

and the formula stated in the theorem follows.

LEMMA 1 If the transverse closed-orbit displacement $\Delta_{\perp}$ of the beam from the magnet center is much larger than the rms beam size $\sqrt{\beta_{\perp} \epsilon_{\perp}}$, the deflection produced by the longitudinal component of the fringe field at one magnet end is

$$
\left\langle\frac{\left|\Delta p_{\|}\right|}{\left|\Delta p_{0}\right|}\right\rangle \approx \frac{\Delta_{\perp}}{L} \sqrt{\frac{\epsilon_{\perp}}{\beta_{\perp}}} \sqrt{\frac{1+0.25 \beta^{\prime 2}}{2}} \approx \frac{\Delta_{\perp}}{L} \sqrt{\frac{\epsilon_{\perp}}{\beta_{\perp}}} .
$$

LEMMA 2 If the transverse displacement of the particle trajectory from the magnet center is the same at the two ends of the magnet, the sum of the deflection produced by the longitudinal component of the fringe field at the two ends is equal to zero. 
Since a typical transverse emittance is $\epsilon_{\perp} \approx 10^{-7} \mathrm{~m}$ and a typical magnet length is $L \approx 1 \mathrm{~m}$, it can be seen that $\left\langle\left|\Delta p_{\|}\right| /\left|\Delta p_{0}\right|\right\rangle$ is typically an extremely small number. This validates the "standard" approximation in essentially all situations in high energy accelerators.

The theorem by no means implies that fringe fields are unimportant. Especially in the intersection regions where the $\beta$-functions are large and rapidly varying, the deflections due to the transverse components of the fringe field can be significant. What the theorem shows is that the expansion of the fringe field in transverse multipoles - also "standard" - continues to be an excellent approximation even though that is not a priori obvious.

Another implication of the theorem is that $\left\langle\left|\Delta p_{\|}\right| /\left|\Delta p_{0}\right|\right\rangle$ is independent of the $\beta$-function at the magnet end, even though that may seem counterintuitive. The reason for this is that $x_{\perp}$ and $v_{\perp}$, the main factors in $\epsilon_{\perp}$, depend inversely on the $\beta$-function.

Perhaps the "worst case" situation for the theorem occurs at the ends of a quadrupole adjacent to a low- $\beta^{*}$ intersection point (IP). If $\beta^{*}$ is the value of the $\beta$-function at the IP, and $s$ is the distance from the IP to the quadrupole edge, one has $\beta^{\prime} \approx 2 s / \beta^{*}$ and the estimate provided by the theorem is

$$
\left\langle\frac{\left|\Delta p_{\|}\right|}{\left|\Delta p_{0}\right|}\right\rangle \approx \frac{\epsilon_{\perp}}{L} \sqrt{\frac{3}{8}} \frac{s}{\beta^{*}} .
$$

Some typical application: for RHIC, $s \approx 30 \mathrm{~m}, L \approx 3 \mathrm{~m}, \beta^{*}=1 \mathrm{~m}$, $\epsilon_{\perp} \approx 10^{-7} \mathrm{~m}$ and the ratio yielded is about $6 \times 10^{-7}$; for CESR, $s \approx 0.6 \mathrm{~m}$, $L \approx 0.6 \mathrm{~m}, \beta^{*}=0.02 \mathrm{~m}, \epsilon_{\perp} \approx 2 \times 10^{-7} \mathrm{~m}$ and the ratio yielded is about $10^{-5}$. 